\title{
The influence of an inclined rotation axis on solar irradiance variations
}

\author{
R. Knaack ${ }^{1}$, M. Fligge ${ }^{1}$, S. K. Solanki ${ }^{2}$, and Y. C. Unruh ${ }^{3}$ \\ 1 Institute of Astronomy, ETH-Zentrum, 8092 Zürich, Switzerland \\ e-mail: rknaack@astro.phys.ethz.ch; fligge@astro.phys.ethz.ch \\ 2 Max-Planck-Institut für Aeronomie, 37191 Katlenburg-Lindau, Germany \\ e-mail: solanki@linmpi.mpg.de \\ 3 Institute of Astronomy, University of Vienna, Türkenschanzstr. 17, 1180 Vienna, Austria \\ e-mail: ycu@astro.univie.ac.at
}

Received 10 April 2001 / Accepted 5 July 2001

\begin{abstract}
Compared with Sun-like stars, the irradiance variations of the Sun over the solar cycle appear to be relatively small for its average activity level (Lockwood et al. 1992; Radick et al. 1998). It has been proposed that the special position of Earth-based observers in the ecliptic plane may give the impression of a subdued solar photometric variability (Schatten 1993). The aim of the present paper is to examine the influence on irradiance variations of a solar rotation axis inclined towards the observer. A three-component model is used to calculate relative flux variations of a given active-region distribution on the surface of the Sun as a function of inclination and wavelength. Wavelength-dependent intensity spectra are used to describe the contributions of the undisturbed photosphere, sunspots and faculae. The spectra result from models that have successfully been used to reproduce a host of solar data and thus represent realistic estimates of the radiative output from these solar features. We find that an inclined rotation axis increases the total solar irradiance variations maximally by $40 \%$. The most probable value is approximately $6 \%$. This is much less than that suggested by former studies, which were based on simple contrast functions. In the averaged Strömgren filters we estimate a most probable increase of the solar variability of $30 \%$. In addition, we estimate the dependence of the flux in the chromospheric Ca II H\&K lines on inclination. We find that the average chromospheric activity level depends only slightly on the inclination angle. The chromospheric variability of Sun-like stars, however, is significantly affected. Nonetheless, our results indicate that a different average inclination of stellar rotation axes relative to the observer cannot explain the discrepancy between the brightness variations of the Sun and Sun-like stars.
\end{abstract}

Key words. Sun: activity - Sun: sunspots - Sun: faculae, plages - Sun: photosphere - stars: activity

\section{Introduction}

The total irradiance of the Sun exhibits a prominent eleven-year cycle with an amplitude of about $0.1 \%$ (Fröhlich 2000), which is correlated with the appearance and evolution of magnetic active regions on the solar surface. The small imbalance between the excess flux of faculae and the flux deficit of sunspots causes the Sun to be brighter during activity maximum than during activity minimum (Foukal \& Lean 1988). Measurements of stellar Ca II H\&K flux (used as a proxy of chromospheric magnetic activity) and contemporaneous Strömgren $b \& y$ photometry (at $472 \mathrm{~nm}$ and $551 \mathrm{~nm}$, respectively) have revealed that the photometric variability of the Sun is

Send offprint requests to: R. Knaack, e-mail: rknaack@astro.phys.ethz.ch subdued by a factor of about 2-3 compared to Sun-like stars of similar magnetic activity (Lockwood et al. 1997; Radick et al. 1998, henceforth referred to as R98).

This gap cannot be explained by increased spectral irradiance variations of the Sun towards shorter wavelengths. Model calculations by Fligge et al. (1998) indicate that the solar spectral variability in the Strömgren filters does not exceed the total irradiance variations by more than $30 \%$.

The special position of a terrestrial observer, who sees the Sun almost equator-on, combined with the fact that solar active regions are confined to low and intermediate heliographic latitudes has been proposed as another explanation for the discrepancy between the Sun and Sunlike stars (Schatten 1993, henceforth referred to as S93). An observer high above the ecliptic plane would see a 
different distribution of sunspots and faculae on the solar disc since the active regions would appear closer to the limb, which is illustrated in Fig. 1 for two zonal bands.
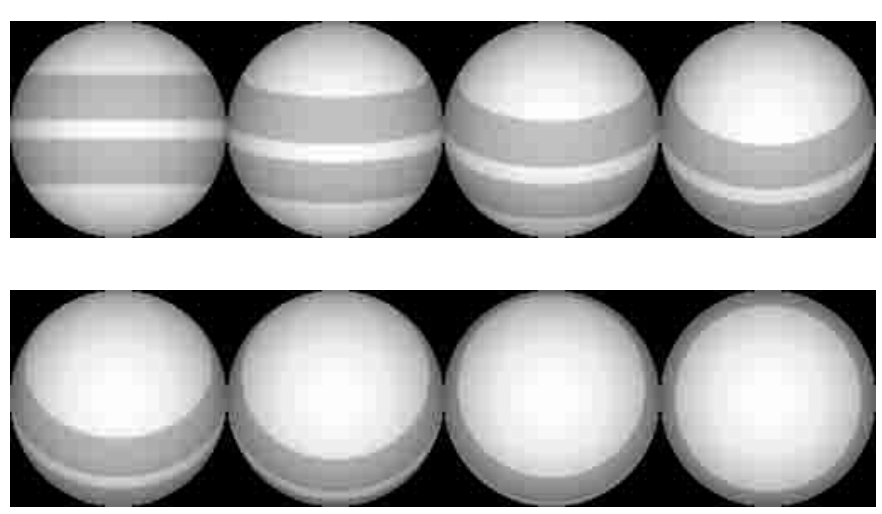

Fig. 1. Visibility of two zonal latitude bands between $\pm 5^{\circ}$ and $\pm 30^{\circ}$ for different inclinations $i$ (defined here as the angle between the solar rotation axis and the line-of-sight); $i$ decreases in steps of $\Delta i \approx 13^{\circ}$ from the upper left $\left(i=90^{\circ}\right)$ to the lower right $\left(i=0^{\circ}\right)$. In this illustration, the contrast of the latitude bands to the rest of the star is taken to be negative and independent of the distance to the limb.

This affects the observed solar irradiance variations because white-light faculae have a higher contrast near the limb than at disc centre while the contrast of sunspots changes only slightly with limb distance. Altogether, a net increase of total irradiance variations is expected when viewing the Sun out of the equatorial plane. Therefore, assuming a random distribution of stellar rotation axes with a most probable inclination of $57^{\circ}$ (R98), this effect tends to enhance the photometric variability of Sun-like stars. At the same time we expect the apparent activity level, as judged by the star's Ca II H\&K flux, to decrease with decreasing inclination $i$ since the Ca II H\&K intensity contrast is roughly independent of limb-distance and the projected area of the active-region bands decreases with $i$ (cf. Fig. 1).

S93 placed active regions as individual dark and bright features, representing sunspots and faculae, on the surface of an artificial Sun in order to investigate the influence of an inclined rotation axis on solar (stellar) irradiance variations. The centre-to-limb variations of the quiet Sun and the active regions were described by quadratic contrast functions adopted from Sofia et al. (1982). The initial variability of the total solar irradiance was set to $0.18 \%$, which yielded a ratio of 5:4 for the contributions from faculae and sunspots. Then, the flux variations for several inclinations were calculated. In the most probable case (i.e. at $i=57^{\circ}$ ), S93 obtained an increase of the total solar irradiance variability by roughly a factor of 3 , which would be sufficient to explain the difference between the Sun and Sun-like stars in the sample of Lockwood et al. (1992).

However, R98 repeated the computations using the same contrast functions, but a more appropriate cyclic variation of $0.10 \%$ (Fröhlich 2000). Moreover, they applied a different ratio of $2: 1$ for the flux contribution of faculae relative to sunspots and an active-region distribution that was completely smeared out over latitude bands. In contradiction to the results of S93, R98 obtained a most probable increase of a factor of only 1.36, which would make the inclination effect of only marginal importance for explaining the observed difference between the Sun and Sun-like stars.

In the following sections, we provide further evidence that the impact of an inclined rotation axis on total solar irradiance variations is small (even smaller than found by R98) and hence not likely to explain the discrepancy between the Sun and Sun-like stars. We improve on the earlier investigations in two ways. Firstly, we make use of empirical atmospheric models to describe the quiet Sun, sunspot and facular components, which is in contrast to the quadratic contrast functions used by S93 and R98. The models have successfully been used to reproduce measurements of facular contrasts (Unruh et al. 1999) as well as total and spectral irradiance variations (Fligge \& Solanki 2000; Fligge et al. 2000a,b). Secondly, we explicitly consider the spectra of each of these components so that we are able to model the fluxes at different wavelengths, e.g. in the Strömgren $b \& y$ filter-bands used for stellar observations.

In addition, we consider the dependence of the Ca II H\&K activity level and variability on the inclination angle. A relative shift between the activity levels of the Sun and Sun-like stars could also lead to a seeming discrepancy between their brightness variations since stellar brightness variability depends on the level of activity (R98). We find that the average activity level only marginally depends on $i$ whereas the cyclic Ca II flux variation between activity minimum and maximum is significantly affected.

Our model is described in detail in Sect. 2. In order to verify our model assumptions, we reproduced the results of S93 and R98. This is shown in Sect. 3. New model calculations for the total irradiance of a star identical to the Sun seen at different $i$ as well as the results for selected wavelength regions and $\mathrm{Ca}$ II H\&K flux are presented in Sect. 4. Finally, the results are summarized and our conclusions are given in Sect. 5 .

\section{Method}

The model consists of three components, namely quietSun, faculae and spots. The intensity spectrum for each of these components are described in Sect. 2.1. Using a slightly modified part of a Doppler-imaging code written by Cameron (1997), we calculated the relative flux variations produced by a given distribution of faculae and spots on the surface of the Sun as a function of inclination of the solar rotation axis and wavelength. The program first computed the visibility of the active-region bands on the solar disc for a given inclination $i$. Then each pixel of the solar surface was replaced by its corresponding intensity 
value according to the analyzed wavelength range, its position on the disc (limb distance) and the component (i.e. quiet Sun, faculae and sunspots) it belonged to. Finally, the flux was calculated by integration over the whole disc.

Our analysis of the inclination effect hence depends on two basic factors. Firstly, the intensity spectra for the individual components and secondly, the geometric configuration of the magnetic features on the solar surface.

\subsection{Intensity spectra}

The intensity spectra were calculated for each component individually as a function of wavelength and limb distance under the assumption of LTE, using Kurucz' spectral synthesis code ATLAS9 (Kurucz 1992). The code employs opacity distribution functions and computes the spectrum from $10 \mathrm{~nm}$ to $160 \mu \mathrm{m}$ with variable resolution. However, we did not consider wavelengths below $160 \mathrm{~nm}$ since the discrepancies between the calculated and observed solar spectrum become prohibitively large in this extreme UV region (Unruh et al. 1999).

The quiet-Sun intensities were obtained with Kurucz' standard solar model atmosphere of $T_{\text {eff }}=5777 \mathrm{~K}$. The spot intensities (averaged over umbra and penumbra) were calculated from a model atmosphere of $5150 \mathrm{~K}$ which in turn was interpolated from the Kurucz grid of model atmospheres. This temperature corresponds to a ratio of umbral to penumbral area of approximately 1:3. Model P of Fontenla et al. (1993) was used as a starting point for the facular model. Slightly modified, this model has been remarkably successful in reproducing the spectral irradiance variations obtained by VIRGO (Fligge et al. 2000a; Fligge et al. 2000b). A detailed description of the models and the resulting spectra can be found in Unruh et al. (1999).

The brightness of active regions relative to the undisturbed photosphere is expressed in terms of the contrast $C_{\mu, \Lambda}$, which depends on the wavelength range $\Lambda$ and on the limb distance parameterized by $\mu=\cos \theta$, with $\theta$ being the angle between the line-of-sight and the normal to the Sun's surface. The contrast is defined by

$C_{\mu, \Lambda}^{\mathrm{f}, \mathrm{s}}=\frac{\int_{\Lambda} I_{\mu}^{\mathrm{f}, \mathrm{s}}(\lambda) \mathrm{d} \lambda}{\int_{\Lambda} I_{\mu}^{\mathrm{qS}}(\lambda) \mathrm{d} \lambda}-1, \quad \mu \in[0,1], \lambda \in \Lambda$.

The superscripts $f$ and $s$ refer to facular and sunspot intensities, respectively, while $I_{\mu}^{\mathrm{qs}}$ stands for the intensity spectrum of the quiet Sun. The computed facular and spot contrasts for the total wavelength range $\Lambda=[160 \mathrm{~nm}, 160 \mu \mathrm{m}]$ as well as for selected passbands are shown in Fig. 2.

Compared to the contrast functions applied by S93 and R98, our spot contrast remains practically independent of $\mu$ for the total wavelength range (Fig. 2a). Note that foreshortening effects due to the Wilson depression were neglected since we do not model individual active regions but active-region bands which are completely smeared out (cf. Sect. 2.2). We do not expect this simplification to significantly influence the results since further investigations showed that the gradient with respect to $\mu$
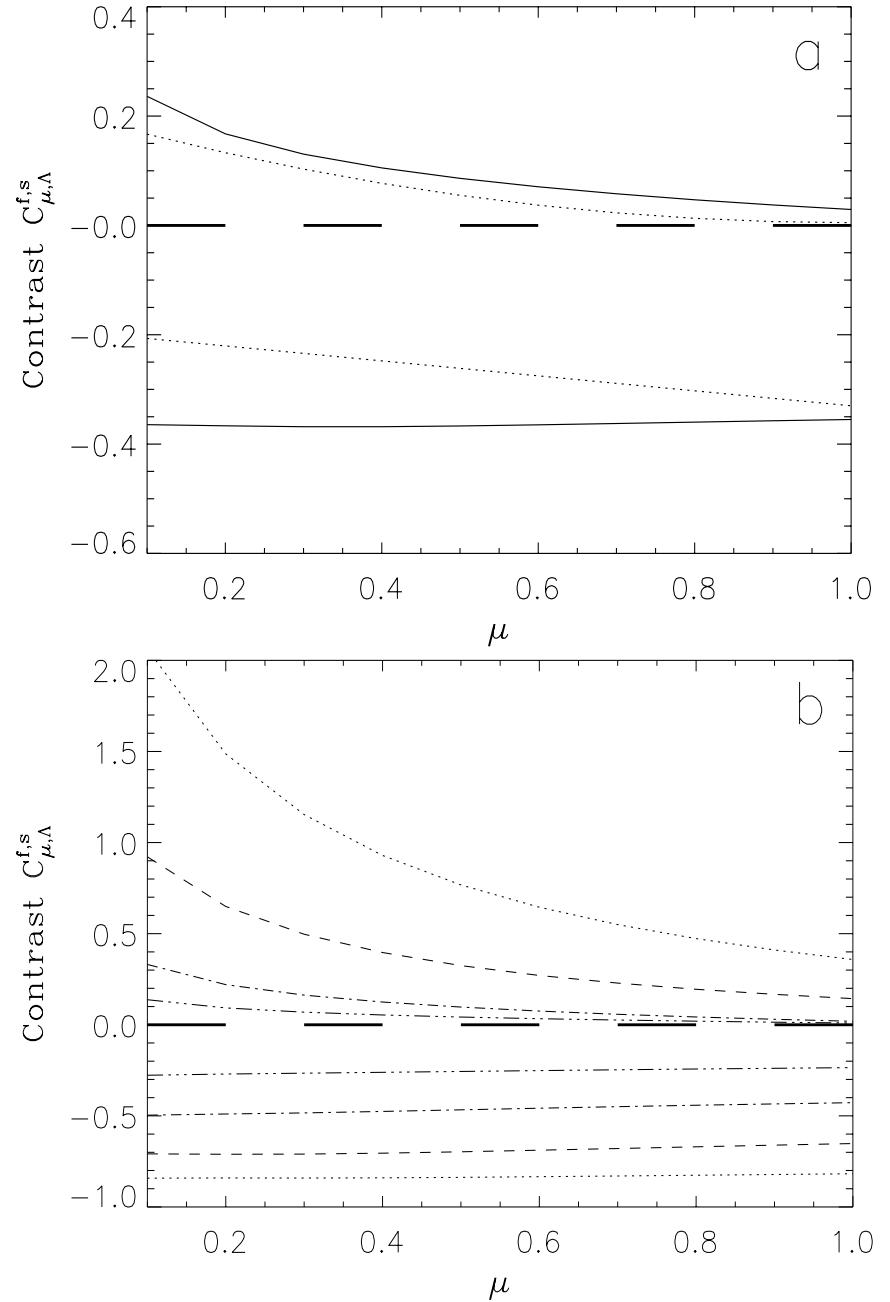

Fig. 2. Facular contrast $\left(C_{\mu, \Lambda}^{\mathrm{f}}>0\right)$ and spot contrast $\left(C_{\mu, \Lambda}^{\mathrm{s}}<0\right)$ vs. $\mu$. Panel a) shows our contrasts for the total wavelength range $\Lambda=[160 \mathrm{~nm}, 160 \mu \mathrm{m}]$ (solid lines) compared to the contrast functions employed by S93 and R98 (dotted). Panel b) shows the contrasts for the wavelength ranges [160 nm, $300 \mathrm{~nm}]$ (dotted), [300 nm, $400 \mathrm{~nm}]$ (dashed), [400 nm, $700 \mathrm{~nm}$ ] (dash dotted) and [700 nm, $160 \mu \mathrm{m}]$ (dash triple dotted).

Table 1. Centre-to-limb variation of the facular contrast $C_{\mu, \Lambda}^{\mathrm{f}}$ from $\mu=1$ to $\mu=0.1$ for several wavelength ranges $\Lambda$, calculated with our facular model. The increase is most accentuated in the visible. The corresponding values for the model given by Sofia et al. (1982) and used by S93 and R98 are given in the last row $(\lambda=500 \mathrm{~nm})$.

\begin{tabular}{cllr}
\hline \hline$\Lambda$ & & & \\
& $C_{\mu=1, \Lambda}^{\mathrm{f}}$ & $C_{\mu=0.1, \Lambda}^{\mathrm{f}}$ & ratio \\
\hline & & & \\
$160 \mathrm{~nm}<\lambda<300 \mathrm{~nm}$ & 0.36 & 2.06 & 5.7 \\
$300 \mathrm{~nm}<\lambda<400 \mathrm{~nm}$ & 0.14 & 0.92 & 6.6 \\
$400 \mathrm{~nm}<\lambda<700 \mathrm{~nm}$ & 0.02 & 0.33 & 16.5 \\
$700 \mathrm{~nm}<\lambda<160 \mu \mathrm{m}$ & 0.01 & 0.13 & 13.0 \\
$160 \mathrm{~nm}<\lambda<160 \mu \mathrm{m}$ & 0.03 & 0.24 & 8.0 \\
Sofia et al. $(1982)$ & 0.005 & 0.167 & 33.4 \\
& & & \\
\hline
\end{tabular}


of the spot contrast only marginally influences the relative flux variations. However, even small changes of the facular contrast can have a strong impact on the results (cf. Sect. 4).

\subsection{Active-region distribution}

The model was calibrated in order to reproduce the behaviour of the Sun during the last two activity cycles, i.e. cycles 21 and 22 . We placed in each solar hemisphere a facular and a sunspot belt, each completely smeared in longitude and latitude within a given latitude band. The magnetic filling factor for each belt was chosen to be in agreement with observations of the total spot and facular coverage of the solar disc at activity maximum and minimum, respectively. At minimum, we set the total area of spots and faculae to zero. Therefore, our analysis does not allow modulations of solar irradiance due to the solar rotation to be investigated but is restricted to long-term changes between activity minimum and maximum.

Recordings of the total spot area $A_{\mathrm{s}}$ during solar cycle 22 suggest a value of $A_{\mathrm{s}} \approx 0.2 \%$ at maximum activity (Chapman et al. 1997). Butterfly diagrams for the same period show that sunspots were mainly confined to a band between $5^{\circ}$ and $30^{\circ}$ on both hemispheres. We hence fixed our sunspot bands accordingly and allowed the associated facular bands to range between $5^{\circ}$ and $40^{\circ}$. In order to achieve an irradiance increase of $0.1 \%$ (Fröhlich 2000) between activity minimum and maximum at an inclination $i=90^{\circ}$, the facular area $A_{\mathrm{f}}$ had to be set to $A_{\mathrm{f}}=2.8 \%$. This corresponds to a ratio of $A_{\mathrm{f}} / A_{\mathrm{s}}=14$, which is in good agreement with observations by Chapman et al. (1997).

Disc integration of the intensities over all visible surface elements finally yielded the total flux $F_{\text {tot }}$ of the Sun at activity maximum as a function of $i$ :

$F_{\text {tot }}(i)=$

$\int_{-1}^{+1} \int_{-\pi / 2}^{+\pi / 2}\left\{\alpha_{\mathrm{f}}(x, \phi, i) I_{\mathrm{f}}(x, \phi)+\alpha_{\mathrm{s}}(x, \phi, i) I_{\mathrm{s}}(x, \phi)\right.$

$\left.+\left(1-\alpha_{\mathrm{f}}(x, \phi, i)-\alpha_{\mathrm{s}}(x, \phi, i)\right) I_{\mathrm{qs}}(x, \phi)\right\} V(\rho) \mathrm{d} \phi \mathrm{d} x$,

where $x=\cos \vartheta, \vartheta$ being the colatitude, $\phi$ is the longitude, $V(\rho)=\sqrt{1-\rho^{2}}=\sin \vartheta \cos \phi$ is the projection factor and $\rho=\left(\cos ^{2} \vartheta+\sin ^{2} \phi \sin ^{2} \vartheta\right)^{\frac{1}{2}}$ is the fractional radius of a given point $(x, \phi)$ on the solar image. Indices $\mathrm{f}, \mathrm{s}$ and qs again refer to faculae, spots and the quiet Sun. The intensities $I$ are integrated over the considered wavelength range $\Lambda$, thus $I(x, \phi)=\int_{\Lambda} I(x, \phi, \lambda) \mathrm{d} \lambda$. The distribution of faculae and spots at a given inclination $i$, i.e. the coverage of a surface element at $(x, \phi, i)$ by the facular or spot component, is determined by the corresponding filling factors $\alpha_{\mathrm{f}}$ and $\alpha_{\mathrm{s}}\left(0 \leq \alpha_{\mathrm{f}}+\alpha_{\mathrm{s}} \leq 1\right)$. Hence, the total areas $A_{\mathrm{f}}$ and $A_{\mathrm{s}}$ are given according to $A_{\mathrm{f}, \mathrm{s}}=\frac{1}{2} \int_{-1}^{+1} \alpha_{\mathrm{f}, \mathrm{s}}\left(x, i=90^{\circ}\right) \mathrm{d} x$. The flux of the Sun at activity minimum (when $\alpha_{\mathrm{f}, \mathrm{s}} \equiv 0$ ) is given by

$F_{\mathrm{qs}}=\int_{-1}^{+1} \int_{-\pi / 2}^{+\pi / 2} I_{\mathrm{qs}}(x, \phi) V(\rho) \mathrm{d} \phi \mathrm{d} x$.
Note that the contribution of the network to the flux is included in $I_{\mathrm{qs}}$. The relative flux variation between activity maximum and minimum is then defined as

$\Delta F_{\mathrm{tot}}(i)=\frac{F_{\mathrm{tot}}(i)-F_{\mathrm{qs}}}{F_{\mathrm{qs}}}$

with $\Delta F_{\text {tot }}\left(90^{\circ}\right)=0.1 \%$. It is useful to introduce $F_{\mathrm{f}, \mathrm{s}}$ and $\Delta F_{\mathrm{f}, \mathrm{s}}$ which analogously describe the contributions of faculae and sunspots:

$\begin{aligned} F_{\mathrm{f}, \mathrm{s}}(i)= & \int_{-1}^{+1} \int_{-\pi / 2}^{+\pi / 2}\left\{\alpha_{\mathrm{f}, \mathrm{s}}(x, \phi, i) I_{\mathrm{f}, \mathrm{s}}(x, \phi)\right. \\ & \left.+\left(1-\alpha_{\mathrm{f}, \mathrm{s}}(x, \phi, i)\right) I_{\mathrm{qS}}(x, \phi)\right\} V(\rho) \mathrm{d} \phi \mathrm{d} x\end{aligned}$

$\Delta F_{\mathrm{f}, \mathrm{s}}(i)=\frac{F_{\mathrm{f}, \mathrm{s}}(i)-F_{\mathrm{qs}}}{F_{\mathrm{qs}}}$.

The visibility of the active-region bands and the associated flux were computed for several inclination angles $i \leq 90^{\circ}$ (cf. Fig. 1) yielding $\Delta F_{\text {tot }}(i)$, which is presented in Sect. 4. The dependence of the projected areas $a_{\mathrm{f}, \mathrm{s}}$ of faculae and spots on $i$, thus $a_{\mathrm{f}, \mathrm{s}}(i)=\frac{1}{\pi} \int_{-1}^{+1} \int_{-\pi / 2}^{+\pi / 2} \alpha_{\mathrm{f}, \mathrm{s}}(x, \phi, i) V(\rho) \mathrm{d} \phi \mathrm{d} x$, is shown in Fig. 3.
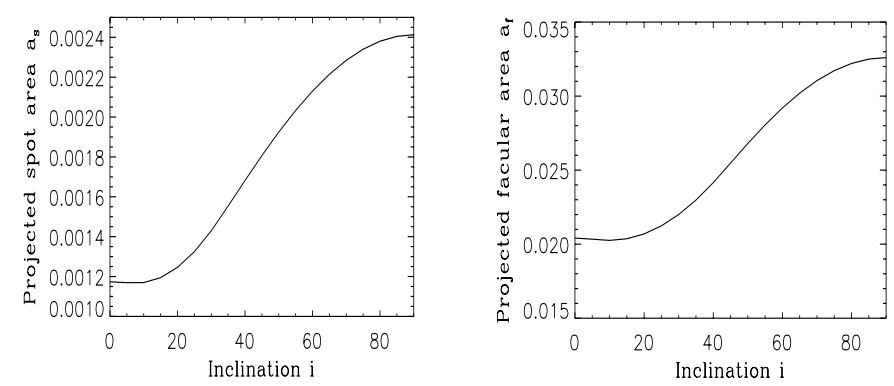

Fig. 3. Dependence of the projected area $a$ on the inclination $i$ of the solar rotation axis for spots $\left(a_{\mathrm{s}}\right.$, left panel) and faculae $\left(a_{\mathrm{f}}\right.$, right panel). The spot belts range between $\delta_{\min }= \pm 5^{\circ}$ and $\delta_{\max }= \pm 30^{\circ}$ and are filled corresponding to a total (not projected) area $A_{\mathrm{s}}=0.20 \%$. The values for the faculae are $\delta_{\text {min }}= \pm 5^{\circ}, \delta_{\max }= \pm 40^{\circ}$ and $A_{\mathrm{f}}=2.80 \%$. The relative loss of area from $i=90^{\circ}$ (equator-on) to $i=0^{\circ}$ (pole-on) is more accentuated for the spots $(-51 \%)$ than for the faculae $(-37 \%)$ due to the wider spread of the facular distribution.

\subsection{Ca I/ flux}

Disc-integrated chromospheric Ca II H\&K line emission is widely used as a proxy of solar and stellar magnetic activity (Skumanich et al. 1975; Wilson 1978; Schrijver et al. 1989). The underlying assumption is that the summed H\&K flux reflects the amount of nonthermal chromospheric heating above faculae, both in active regions and network, which in turn is associated with surface magnetic fields. An instrumental index of the Ca II H\&K flux is the $S$-index, which is proportional to the summed $\mathrm{H}$ and $\mathrm{K}$ 
fluxes normalized by the summed fluxes of two nearby continuum bands (see R98 for details).

As discussed in Sect. 4.3, the Ca II flux also depends on inclination. Hence, we investigated $S$ as a function of $i$. Measurements of Skumanich et al. (1984) indicate that the facular intensity at the solar Ca K line centre darkens the same way as the quiet-Sun intensity with decreasing $\mu$. Since our intensity spectra were calculated under the assumption of LTE, they do not describe the Ca II H\&K line cores accurately. Thus we adopted $I_{\mathrm{qs}}(\mu)$ from Skumanich et al. (1984).

Under the explicit assumption that the facular contrast in the Ca II H\&K lines remains constant over the disc, i.e.

$\frac{I_{\mathrm{f}}(\mu)}{I_{\mathrm{qs}}(\mu)}=$ const. $\equiv \frac{S_{\mathrm{f}}}{S_{\mathrm{qs}}}$,

one can roughly estimate the $i$-dependence of the solar $S$-index at activity maximum as

$S_{\mathrm{tot}}(i)=\tilde{\alpha}_{\mathrm{f}}(i) S_{\mathrm{f}}+\left(1-\tilde{\alpha}_{\mathrm{f}}(i)\right) S_{\mathrm{qs}}$,

where the geometric distribution of the faculae is included in

$\tilde{\alpha}_{\mathrm{f}}(i)=\frac{\int_{-1}^{+1} \int_{-\pi / 2}^{\pi / 2} \alpha_{\mathrm{f}}(x, \phi, i) I_{\mathrm{qS}}(x, \phi) V(\rho) \mathrm{d} \phi \mathrm{d} x}{\int_{-1}^{+1} \int_{-\pi / 2}^{\pi / 2} I_{\mathrm{qs}}(x, \phi) V(\rho) \mathrm{d} \phi \mathrm{d} x}$.

Thus, $S$ is written as the weighted sum of a facular contribution $S_{\mathrm{f}}$ (which is the $S$-index of an artificial Sun completely covered with faculae) and a quiet-Sun contribution $S_{\mathrm{qs}}$. Both are independent of $i . S_{\mathrm{tot}}\left(i=90^{\circ}\right)$ and $S_{\mathrm{qs}}$ can be derived from observations of the solar Ca II K line profile at maximum and minimum activity, respectively. $S_{\mathrm{f}}$ is then determined by Eq. (8).

The Ca II K line profile has been measured by two independent programs conducted at the Sac Peak Evans' Facility and the Kitt Peak McMath-Pierce telescope. We used the daily Sac Peak $K$-index $\left(K_{\mathrm{SP}}\right)$, which can be adjusted to the monthly Kitt Peak $K$-index $\left(K_{\mathrm{KP}}\right)$ by $K_{\mathrm{KP}}=-0.01+1.1 K_{\mathrm{SP}}$ (White et al. 1998). The transformation between $K_{\mathrm{KP}}$ and $S$ is given by R98 as $S=$ $0.041+1.475 K_{\mathrm{KP}}$. We thus obtained $S_{\mathrm{qs}}=0.170$ for the minimum in 1986 (which is close to the value of 0.169 suggested by R98) and $S_{\text {tot }}\left(i=90^{\circ}\right)=0.187$ for the maximum in 1990. These values imply that $S_{\mathrm{f}}=0.675$. The dependence of $S_{\text {tot }}$ on $i$ is presented in Sect. 4.3.

\section{Reproduction of earlier results}

We first reproduced the results of S93 and R98 for the total wavelength range in order to verify our code and to separate the influence of the different assumptions made in each approach. The main distinction between these earlier investigations and the present analysis is the origin of the contrast functions (cf. Fig. 2). S93 and R98 used the active-region model of Sofia et al. (1982), where the limbdarkening of the undisturbed photosphere and the contrast of spots and faculae are described by second degree polynomials.
In contrast, our calculations employ intensity spectra for faculae, spots and the quiet Sun as described in Sect. 2.1. Moreover, we explicitly fix the spot area $A_{\mathrm{s}}$ according to observations and tune the facular area $A_{\mathrm{f}}$ (and hence the relative flux variation $\Delta F_{\mathrm{f}}\left(90^{\circ}\right)$ ) in order to obtain a cyclic variation of $\Delta F_{\text {tot }}\left(90^{\circ}\right)=0.1 \%$. Consequently, $A_{\mathrm{f}}$ and $\Delta F_{\mathrm{f}, \mathrm{s}}\left(90^{\circ}\right)$ are outputs of our model and can hence be compared to observations.

The models also differ in the assumed geometric distribution of the active regions. S93 placed individual dark and bright features on the solar surface while R98 used smeared distributions, which is similar to our procedure.

\subsection{Model calculations according to $S 93$}

S93 placed the individual groups of spots and faculae within two bands ranging from $10^{\circ}$ to $40^{\circ}$ on each hemisphere. The areas were adjusted to provide a cyclic variation of about $0.18 \%$, which is in reasonable agreement with the results of the uncorrected ERB radiometer on Nimbus 7 (Kyle et al. 1994) but almost a factor of 2 larger than the currently accepted value. Besides, the numbers of S93 imply an (unsigned) ratio of facular-to-spot flux variation of $\Delta F_{\mathrm{f}}\left(90^{\circ}\right) \Delta F_{\mathrm{s}}\left(90^{\circ}\right)=54$.

The original results of S93 are shown in Fig. 4a. The relative flux variation $\Delta F_{\text {tot }}$ (solid line) starts at $0.18 \%$ $\left(i=90^{\circ}\right)$ and increases up to $1.1 \%\left(i=30^{\circ}\right)$, i.e. by a factor of 6 , followed by a pronounced decline. For the statistically most probable case of $i=57^{\circ}$, the irradiance increases by a factor of about 3 . The dotted line shows the contribution of spots $\Delta F_{\mathrm{s}}$ and the dashed line the contribution of faculae $\Delta F_{\mathrm{f}}$.

Our reproduction under the same conditions is shown in Fig. 4b. Instead of our intensity spectra we hence used the polynomials proposed by Sofia et al. (1982), i.e. the same limb-darkening function for the undisturbed photosphere, the same contrast functions for the active regions and finally a similar geometry like the one applied by S93 (although we used smeared latitude bands). It turned out that it was necessary to employ very large facular and spot areas to fulfil the boundary conditions of S93 (i.e. $\Delta F_{\text {tot }}=0.18 \%$ and $\Delta F_{\mathrm{f}}: \Delta F_{\mathrm{s}}=5: 4$ at $i=90^{\circ}$ ), namely $A_{\mathrm{f}}=34 \%$ and $A_{\mathrm{s}}=2.4 \%$. This is a factor of about 10 larger than observations of the active Sun imply. In agreement with S93, we then obtained a maximal increase between $i=90^{\circ}$ and $i=0^{\circ}$ of a factor of 6 while the value for the most probable inclination was about 2 . As can be seen, however, there is a substantial difference between the result of S93 and our reproduction regarding the dependence of the flux variation on inclination, which is probably due to the different distributions applied.

In a next step we replaced the limb-darkening function of the quiet Sun and the contrasts for spots and faculae by our intensity spectra. However, the relative flux variations at $i=90^{\circ}$ were kept, i.e. $\Delta F_{\text {tot }}=0.18 \%$ and $\Delta F_{\mathrm{f}}: \Delta F_{\mathrm{s}}=5: 4$. This reduced the necessary total facular and spot areas to $A_{\mathrm{f}}=14 \%$ and $A_{\mathrm{s}}=1.9 \%$, which is still 

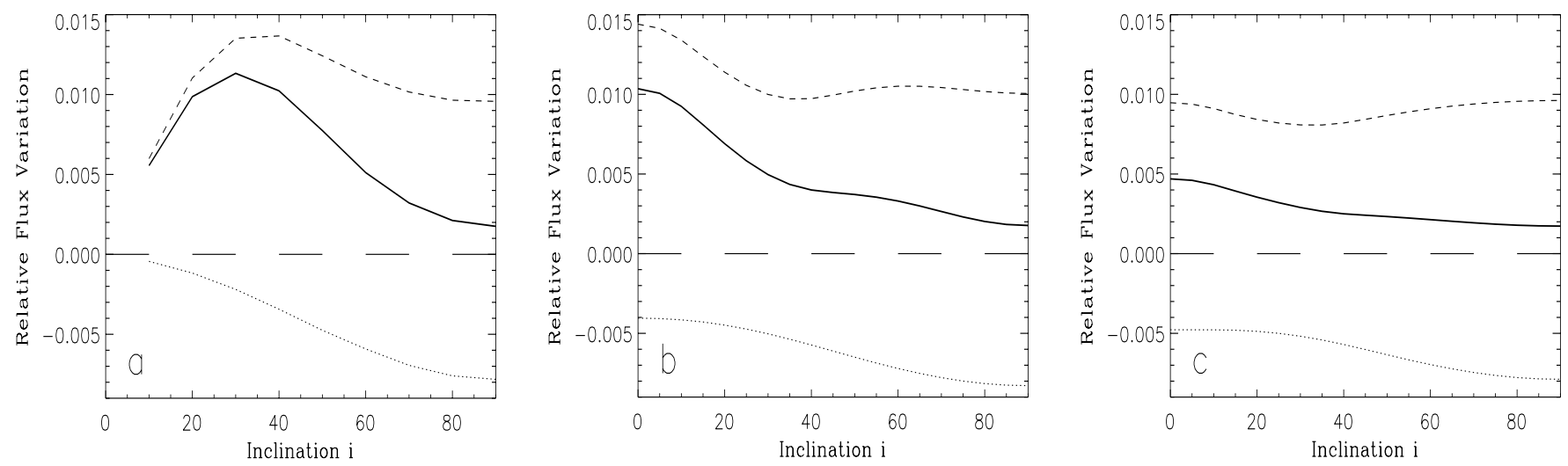

Fig. 4. Relative variation $\Delta F_{\text {tot }}$ (bold line) of the total flux vs. inclination $i$ and the contributions from faculae $\Delta F_{\mathrm{f}}$ (dashed) and spots $\Delta F_{\mathrm{S}}$ (dotted). Panel a) shows the original results of S93. He applied an initial variation of $\Delta F_{\text {tot }}\left(90^{\circ}\right)=0.18 \%$, a ratio of $\Delta F_{\mathrm{f}}\left(90^{\circ}\right): \Delta F_{\mathrm{s}}\left(90^{\circ}\right)=5: 4$ and limb-darkening and contrast functions described in the text. Panel b) shows our reproduction using the same boundary conditions (i.e. the same $\Delta F_{\text {tot }}$ and $\Delta F_{\mathrm{f}}: \Delta F_{\mathrm{s}}$ at $i=90^{\circ}$ ), the same limb-darkening function and contrasts but a slightly different distribution of the active regions. The required (facular and spot) areas were $A_{\mathrm{f}}=34 \%$ and $A_{\mathrm{s}}=2.4 \%$, which is a factor 10 larger than present observations imply. Panel c) was calculated again under the same boundary conditions but this time by replacing the limb-darkening and contrast functions with the spectra of our model. The required areas were $A_{\mathrm{f}}=14 \%$ and $A_{\mathrm{s}}=1.9 \%$.

too high but significantly less than before. The result is shown in Fig. 4c. The variation increases now maximally by a factor of 2.7 , in the most probable case only by a factor of 1.3. Our facular and sunspot models hence predict a much smaller inclination effect, even if we employ the cyclic variation of $0.18 \%$.

\subsection{Model calculations according to R98}

R98 applied the same limb-darkening and contrast functions as S93. However, R98 used a cyclic irradiance variation of $\Delta F_{\text {tot }}\left(90^{\circ}\right)=0.1 \%$ and a facular-to-spot flux variation of $\Delta F_{\mathrm{f}}: \Delta F_{\mathrm{s}}=2: 1\left(i=90^{\circ}\right)$. The sunspot belts were confined to a band between $15^{\circ}$ and $30^{\circ}$ in each hemisphere. The facular belts ranged between $5^{\circ}$ and $40^{\circ}$. Similar to our model, the active-region bands were smeared completely in longitude. Under these conditions they found a most probable cyclic variation of $0.136 \%$ (at $\left.i=57^{\circ}\right)$ and a maximum increase of a factor of about 2 (at $i=90^{\circ}$ ).

Again we reproduced their results by employing the same boundary conditions and the same limb-darkening and contrast functions. Because of the similar geometry we expected better accordance this time. Figure 5 shows our reproduction for $A_{\mathrm{f}}=7 \%$ and $A_{\mathrm{s}}=0.28 \%$. We calculated a most probable variation of $\Delta F_{\text {tot }}\left(57^{\circ}\right)=0.133 \%$ and a maximum variation of $\Delta F_{\text {tot }}\left(0^{\circ}\right)=0.231 \%$. This is in good agreement with R98. However, the total facular area of $7 \%$ implies a facular-to-spot ratio of $A_{\mathrm{f}} / A_{\mathrm{s}}=25$ whereas observations by Chapman et al. (1997) suggest a ratio of $A_{\mathrm{f}} / A_{\mathrm{s}} \approx 14$ for the solar maximum.

The disagreement between the prediction of S93 and the one of R98 indicates that the inclination effect strongly depends on the boundary conditions, i.e. the exact values of $\Delta F_{\text {tot }}\left(90^{\circ}\right)$ and the ratio $\Delta F_{\mathrm{f}}\left(90^{\circ}\right): \Delta F_{\mathrm{s}}\left(90^{\circ}\right)$. Our analysis reduced the free parameters (besides the

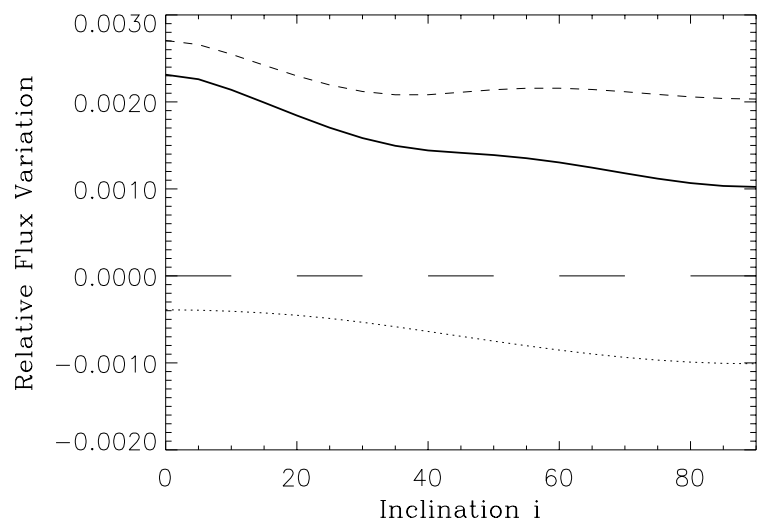

Fig. 5. Reproduction of the inclination effect using the same contrast and boundary conditions as R98, i.e. $\Delta F_{\text {tot }}\left(90^{\circ}\right)=$ $0.10 \%$ and $\Delta F_{\mathrm{f}}\left(90^{\circ}\right): \Delta F_{\mathrm{s}}\left(90^{\circ}\right)=2: 1$. The spot and facular areas were set to $A_{\mathrm{s}}=0.28 \%$ and $A_{\mathrm{f}}=25 A_{\mathrm{s}}$.

geometric distribution) to the total sunspot area $A_{\mathrm{s}}$ and the total irradiance variation $\Delta F_{\text {tot }}\left(90^{\circ}\right)$ (which directly determine the total facular area $A_{\mathrm{f}}$ and the ratio $\left.\Delta F_{\mathrm{f}}\left(90^{\circ}\right): \Delta F_{\mathrm{S}}\left(90^{\circ}\right)\right)$. Both parameters are accessible to modern observations. The boundary conditions of S93 required unrealistically large areas for active regions. The values assumed by $\mathrm{R} 98$ implied a spot area of $A_{\mathrm{s}}=0.28 \%$ and a ratio of $A_{\mathrm{f}} / A_{\mathrm{s}}=25$. While the Sun may have reached such a large $A_{\mathrm{s}}$ value at the maximum of cycle 19 , the most active cycle on record, the facular-to-spot area ratio of 25 is definitely too large for solar maximum so that the $A_{\mathrm{f}}$ value itself is too large.

\section{Results}

In this section we investigate the inclination effect using what we consider to be the most realistic set of parameters. Our model allows the inclination effect to be 
investigated for almost any wavelength range between $160 \mathrm{~nm}$ and $160 \mu \mathrm{m}$. In the following, we first consider the total, wavelength integrated irradiance and then the irradiance in particular passbands.

\subsection{Increase of total irradiance}

We restricted our input parameters to the following observed quantities (cf. Sect. 2.2):

- total spot coverage $A_{\mathrm{s}}=0.20 \%$;

- total irradiance variability of $\Delta F_{\text {tot }}\left(90^{\circ}\right)=0.10 \%$ (which yields a facular area of $A_{\mathrm{f}}=2.80 \%$ and a ratio of $\Delta F_{\mathrm{f}}\left(90^{\circ}\right): \Delta F_{\mathrm{S}}\left(90^{\circ}\right)=2: 1$, the latter is equal to the ratio used by $\mathrm{R} 98)$;

- active regions belts ranging from $5^{\circ}$ to $30^{\circ}$ for spots and from $5^{\circ}$ to $40^{\circ}$ for faculae.

The obtained area ratio of $A_{\mathrm{f}} / A_{\mathrm{s}}=14$ for solar activity maximum is in good agreement with observations (Chapman et al. 1997). The resulting inclination effect is shown in Fig. 6.

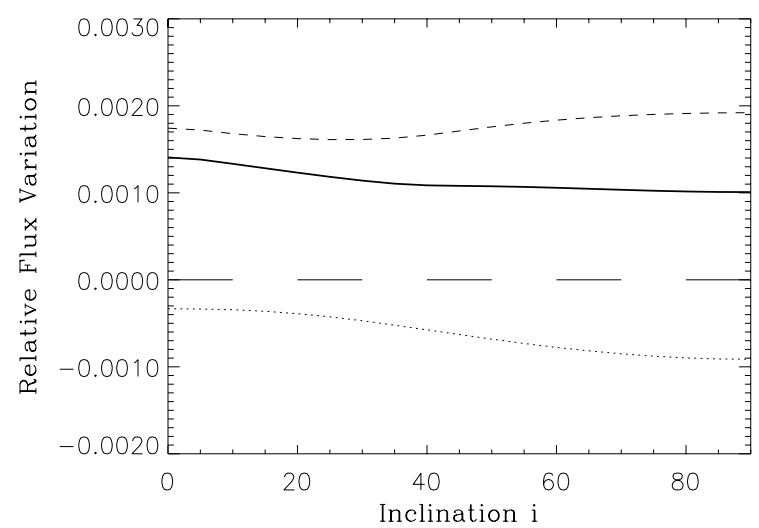

Fig. 6. Relative variation $\Delta F_{\text {tot }}$ (bold line) of the total flux vs. inclination $i$ and the contributions from faculae $\Delta F_{\mathrm{f}}$ (dashed) and spots $\Delta F_{\mathrm{s}}$ (dotted).

We have obtained a most probable amplitude for the cyclic variation of $\Delta F_{\text {tot }}\left(57^{\circ}\right)=0.106 \%$ and a maximum amplitude of $\Delta F_{\text {tot }}\left(0^{\circ}\right)=0.141 \%$. These variations are significantly smaller than the values found by R98, which is mainly due to the different centre-to-limb variation (CLV) of the facular contrast of our model (cf. Fig. 2). Using an alternative facular contrast, R98 found a most probable variation of $0.117 \%$, which is closer to our result and underlines the strong influence of the CLV of the facular contrast. We also investigated the influence of varying the input parameters: changes of $10 \%$ in the spot area or the total irradiance variation (at $i=90^{\circ}$ ) resulted in a range from $0.105 \%$ to $0.107 \%$ for the most probable amplitude and from $0.13 \%$ to $0.15 \%$ for the maximum.

Further test calculations showed that changes in the linear spot contrast function hardly affect the gradient of $\Delta F_{\text {tot }}(i)$, whereas changes in the facular contrast have a significant influence. Nevertheless, it is unlikely that a maximum increase exceeding a factor of 1.7 can be obtained for reasonable values of the facular area $A_{\mathrm{f}}$.

\subsection{Wavelength dependence}

Using the same input parameters as in Sect. 4.1, we calculated $\Delta F_{\text {tot }}(i)$ for several Gaussian filters with $\sigma=25 \mathrm{~nm}$ and centered at $\lambda=200,250,300, \ldots, 1000 \mathrm{~nm}$. The relative flux variation $\Delta F_{\text {tot }}$ as a function of wavelength $\lambda$ is shown in Fig. 7a for the inclinations $i=90^{\circ}, i=57^{\circ}$ and $i=0^{\circ}$.
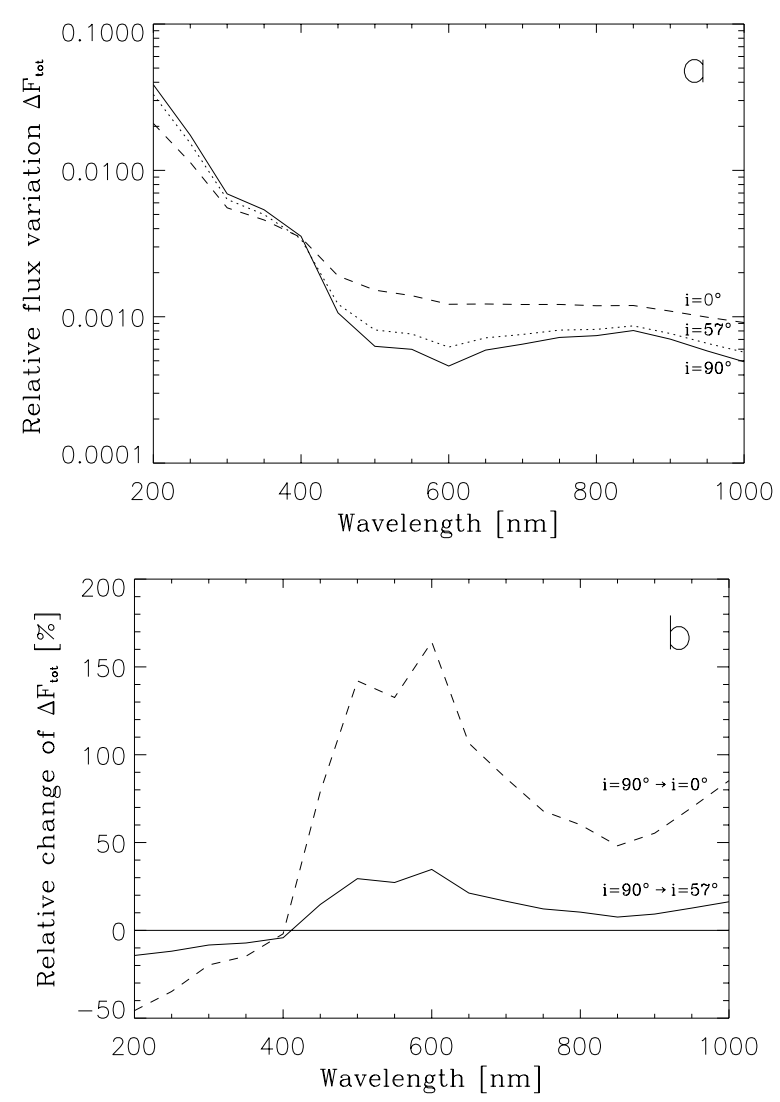

Fig. 7. Relative flux variation $\Delta F_{\text {tot }}$ vs. wavelength for the inclinations $i=90^{\circ}, i=57^{\circ}$ and $i=0^{\circ}$ (panel a)). The relative change of $\Delta F_{\text {tot }}$, thus $\left[\Delta F_{\text {tot }}\left(57^{\circ}\right)-\Delta F_{\text {tot }}\left(90^{\circ}\right)\right] / \Delta F_{\text {tot }}\left(90^{\circ}\right)$ (solid) and $\left[\Delta F_{\text {tot }}\left(0^{\circ}\right)-\Delta F_{\text {tot }}\left(90^{\circ}\right)\right] / \Delta F_{\text {tot }}\left(90^{\circ}\right)$ (dashed), is shown in panel b). The zero line is indicated.

There is a sharp rise in $\Delta F_{\text {tot }}$ for wavelengths smaller than $450 \mathrm{~nm}$ due to the increasing facular variability $\Delta F_{\mathrm{f}}$ in the ultraviolet. Unexpectedly, $\Delta F_{\text {tot }}\left(i=90^{\circ}\right)$ becomes larger than $\Delta F_{\text {tot }}\left(i<90^{\circ}\right)$ for $\lambda \lesssim 400 \mathrm{~nm}$. The irradiance variability hence decreases for smaller values of $i$, which is the opposite to the behaviour at wavelengths longer than $400 \mathrm{~nm}$. This is seen more clearly in Fig. 7b, which shows the change of $\Delta F_{\text {tot }}$ from $i=90^{\circ}$ to $i=57^{\circ}$ and $i=0^{\circ}$, respectively. The reasons for this behaviour are discussed below in conjunction with Fig. 9 .

The passbands of the Strömgren $b \& y$ filters, which are used for the photometric observations of Sun-like stars, are centered at $472 \mathrm{~nm}$ and $551 \mathrm{~nm}$. For the averaged filters, 
i.e. $(b+y) / 2$, we estimate a most probable increase of a factor of roughly 1.3 and a maximum increase of about 2.2. However, the facular contrast in these passbands (and hence $\Delta F_{\mathrm{f}}$ and $\left.\Delta F_{\text {tot }}\right)$ sensitively depends on the exact temperature of the facular model at the relevant atmospheric layers, which is not well determined due to the lack of suitable observations. The values for the variability at the Strömgren wavelengths can therefore only be taken as a first estimate. More reliable estimates must await, for instance, resolved measurements of solar spectral irradiance variations which will allow tighter constraints on the employed facular model.

Finally, we calculated the relative flux variability for the passbands [160 nm, $300 \mathrm{~nm}$ ], [300 nm, $400 \mathrm{~nm}$ ], $[400 \mathrm{~nm}, 700 \mathrm{~nm}]$ and $[700 \mathrm{~nm}, 160 \mu \mathrm{m}]$. The results are shown in Fig. 9. There is again a decrease in $\Delta F_{\text {tot }}$ from $i=90^{\circ}$ to $i=0^{\circ}$ for the filters with $\lambda<400 \mathrm{~nm}$ (panels a and $b$ ).

The reason lies in the CLV of the facular contrast. For $\lambda<400 \mathrm{~nm}$, the increase of $C_{\mu, \Lambda}^{f}$ from disc centre $(\mu=1)$ to the limb $(\mu=0.1)$ is too small to compensate for the reduction in projected facular area (cf. Table 1 and Fig. 2). This results in a monotonicly falling facular variability $\Delta F_{\mathrm{f}}$ from $i=90^{\circ}$ (maximal area) to $i=0^{\circ}$ (minimal area). Since the qualitative behaviour of $\Delta F_{\mathrm{s}}$ does not vary significantly with wavelength, the wavelength dependence of $\Delta F_{\text {tot }}$ is mainly dominated by $\Delta F_{\mathrm{f}}$.

\subsection{Influence of changing inclination on chromospheric activity}

It is important to bear in mind that as $i$ is decreased, Sunlike stars appear less active than they would at $i=90^{\circ}$. The Ca II H\&K emission (used to gauge stellar magnetic activity) of a particular surface element increases with the magnetic flux therein (at least outside sunspots; Schrijver et al. 1989). Thus the Ca II H\&K emission is strongest in faculae. Unlike white-light faculae formed in the photosphere, the chromospheric Ca II radiation shows roughly the same contrast all over the disc (Skumanich et al. 1984). However, the projected area of faculae (or, equivalently, chromospheric plages) decreases with decreasing $i$, at least as long as the faculae are situated near the equator.

For faculae with a total area of $A_{\mathrm{f}}=2.8 \%$ and located within latitude bands from $5^{\circ}$ to $40^{\circ}$, the projected surface area drops from $3.26 \%$ at $i=90^{\circ}$ to $2.04 \%$ at $i=0^{\circ}$, i.e. by $37 \%$ (cf. Fig. 3). Since the quiet Sun also contributes to the measured Ca II H\&K flux, the chromospheric flux is expected to change by a smaller factor with $i$.

Figure 8 shows the $S$-index of the active Sun as a function of $i$ according to Eq. (8). At $i=90^{\circ}, S$ is equal to 0.187 . As $i$ changes from $90^{\circ}$ to $0^{\circ}, S$ drops from 0.187 to 0.179 , i.e. by $4 \%$. From $90^{\circ}$ to $57^{\circ}$, the drop is only $1 \%$. However, the effect is much larger for $\Delta S=S-S_{\mathrm{qs}}$ where $S_{\mathrm{qs}}=0.170$ is the $S$-index of the Sun at activity minimum and hence independent of $i$. From $90^{\circ}$ to $57^{\circ}, \Delta S$ decreases

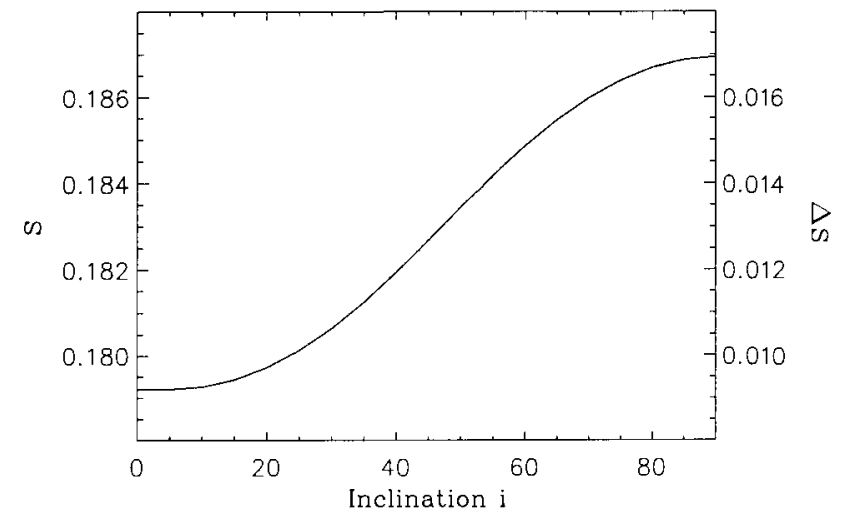

Fig. 8. $S$-index vs. $i$ (left axis) calculated with Eq. (8) for the active Sun and $\Delta S=S-S_{\mathrm{qs}}$ vs. $i$ (right axis). $S_{\mathrm{qs}}=$ 0.170 is the $S$-index of the quiet Sun which is assumed to be independent of $i$.

by $15 \%$, from $90^{\circ}$ to $0^{\circ}$ even by $46 \%$ (i.e. nearly by a factor of 2).

Hence, when comparing stars with similar apparent Ca II H\&K fluxes, we might actually look at stars with different true Ca II H\&K emissions. This bias, again, is introduced by the different inclination angles of the stellar rotation axes. Although the magnitude of the bias is in the range of only a few percent for the absolute flux, it has a significant impact when the flux variation between activity minimum and maximum is considered. Measurements of the temporal Ca II H\&K flux variation thus tend to underestimate the true cyclic chromospheric variability of Sun-like stars.

The $i$-dependence of $\Delta S$ may hence partly be responsible for the dispersion seen in the cyclic chromospheric variation of the sample of Sun-like stars investigated by Lockwood et al. (1992) and R98. However, as explained above, the absolute chromospheric activities are only marginally affected. Therefore, we do not expect that this effect will significantly influence the gap between the broad-band brightness variations exhibited by the Sun and Sun-like stars.

\section{Summary and conclusions}

We have investigated the influence of an inclined rotation axis on the total irradiance variations of the Sun, using atmospheric models to calculate the centre-to-limb variation of the quiet photosphere and the contrasts of faculae and sunspots. These models have already been successfully employed to reproduce time series of solar irradiance on time scales ranging from days up to the length of the solar cycle. We have found that the effects of an inclined rotation axis on solar variability have been overestimated. This is summarized in Fig. 10, which compares our results for the total irradiance with the calculations made with similar choices of the parameters as R98 and S93. The results of both studies could be roughly reproduced by our model, but only under assumptions concerning the employed spot and facular areas that are at best only partly 

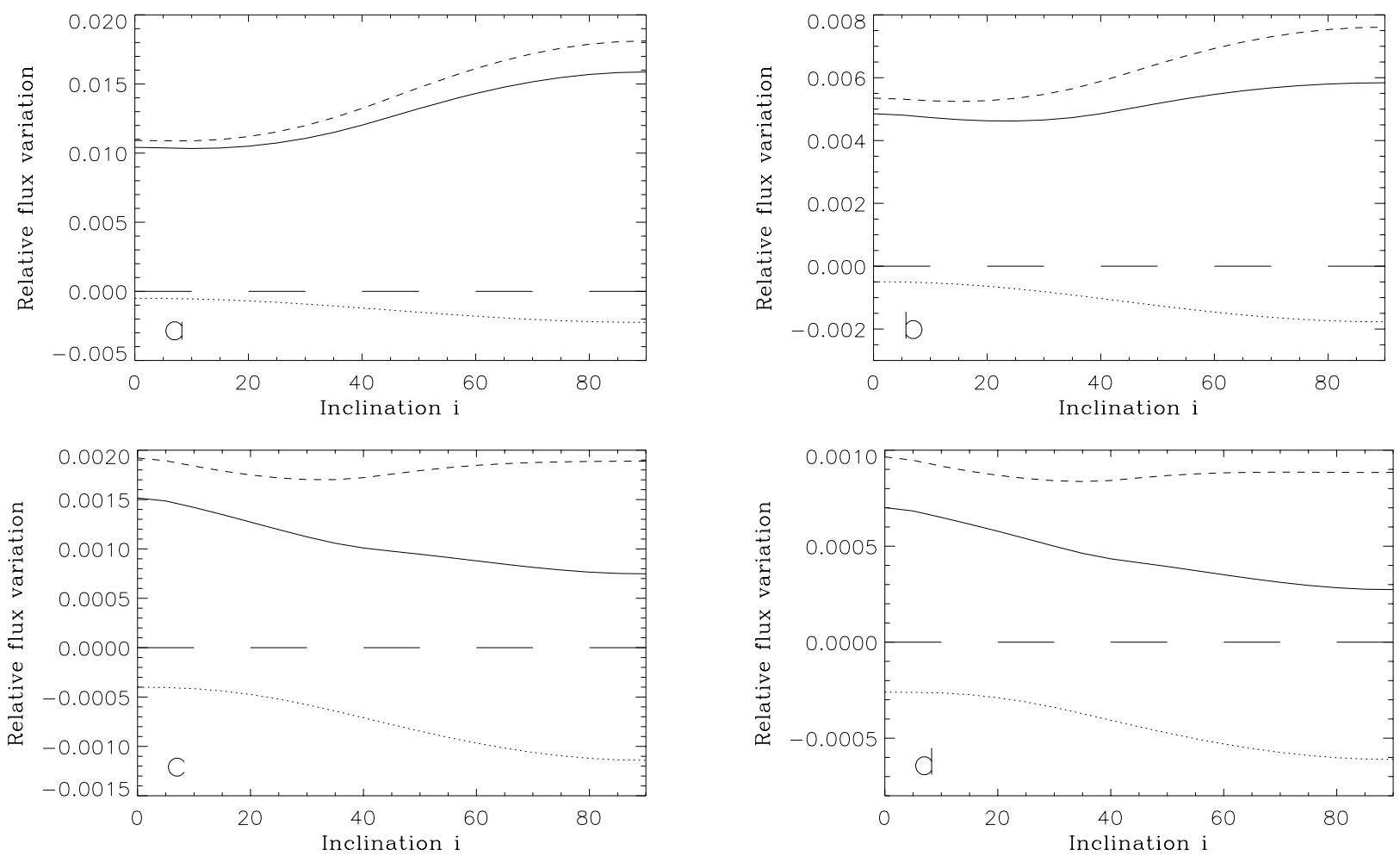

Fig. 9. Relative flux variation $\Delta F_{\text {tot }}$ (solid) vs. inclination $i$ and the contribution of spots $\Delta F_{\mathrm{s}}\left(\right.$ dotted) and faculae $\Delta F_{\mathrm{f}}$ (dashed). Plotted are the results for the four wavelength ranges a) $160 \mathrm{~nm}<\lambda<300 \mathrm{~nm}$, b) $300 \mathrm{~nm}<\lambda<400 \mathrm{~nm}$, c) $400 \mathrm{~nm}<\lambda<700 \mathrm{~nm}$ and d) $700 \mathrm{~nm}<\lambda<160 \mu \mathrm{m}$.

Table 2. Input parameters and computed values (bold) for our model, which used intensity spectra, and for the reproduction of the inclination effect according to S93 and R98, who used contrast functions. Only values referring to the total wavelength range are listed. The distribution of spots and faculae on each hemisphere is given in the second and third column. $A_{\mathrm{s}}$ is the total spot area as per cent of the solar surface, $A_{\mathrm{f}}$ is the same for the faculae. $\Delta F_{\mathrm{f}}: \Delta F_{\mathrm{s}}$ stands for the facular-to-spot flux variability ratio at the inclination $i=90^{\circ},\left(\Delta F_{\text {tot }}\right) \odot$ gives the assumed variation of the Sun over the solar cycle. The factors for the maximum $\left(\right.$ at $i=0^{\circ}$ ) and the most probable increase (at $\left.i=57^{\circ}\right)$ of $\Delta F_{\text {tot }}$, hence $\Delta F_{\text {tot }}\left(0^{\circ}\right) /\left(\Delta F_{\text {tot }}\right) \odot$ and $\Delta F_{\text {tot }}\left(57^{\circ}\right) /\left(\Delta F_{\text {tot }}\right) \odot$, are listed in the last two columns. Values in brackets give the original results found by S93 or R98.

\begin{tabular}{|c|c|c|c|c|c|c|c|c|}
\hline model & spots & faculae & $A_{\mathrm{s}}$ & $A_{\mathrm{f}}$ & $\Delta F_{\mathrm{f}}: \Delta F_{\mathrm{s}}$ & $\left(\Delta F_{\text {tot }}\right)_{\odot}$ & $\max$ & prob \\
\hline Schatten & $10^{\circ}-40^{\circ}$ & $10^{\circ}-40^{\circ}$ & $\mathbf{2 . 4} \%$ & $\mathbf{3 4} \%$ & $5: 4$ & $0.18 \%$ & $6(6)$ & $(3)$ \\
\hline Radick & $15^{\circ}-30^{\circ}$ & $5^{\circ}-40^{\circ}$ & $0.28 \%$ & $7.0 \%$ & $2: 1$ & $0.10 \%$ & $2(2)$ & $1.33(1.36)$ \\
\hline present analysis & $5^{\circ}-30^{\circ}$ & $5^{\circ}-40^{\circ}$ & $0.20 \%$ & $\mathbf{2 . 8} \%$ & $2: 1$ & $0.10 \%$ & 1.4 & 1.06 \\
\hline
\end{tabular}

consistent with observations. The parameters needed for the reproductions are listed in Table 2.

Based on a model that is consistent with observational constraints, we predict an increase of the total solar irradiance variations of $(6 \pm 1) \%$ when decreasing $i$ from $90^{\circ}$ (solar case) to the most probable case of $57^{\circ}$. The predicted maximum increase is $(40 \pm 10) \%$ when decreasing $i$ from $90^{\circ}$ to $0^{\circ}$. Considering the spectral irradiance variations in the Strömgren $b \& y$ filters, we estimate a most probable increase of roughly $30 \%$ for the averaged $(b+y) / 2$ filter. Although the amount of the flux increase depends sensitively on the facular contrast, it appears unlikely that the excessive irradiance variations of other inactive cool stars can be explained by an inclination effect. This is in agreement with the conclusions of R98 and recent observations by Henry (1999).

Moreover, we have found that the Ca II flux variation of Sun-like stars between activity minimum and maximum significantly depends on the inclination of the rotation axis relative to an observer. This result indicates that 


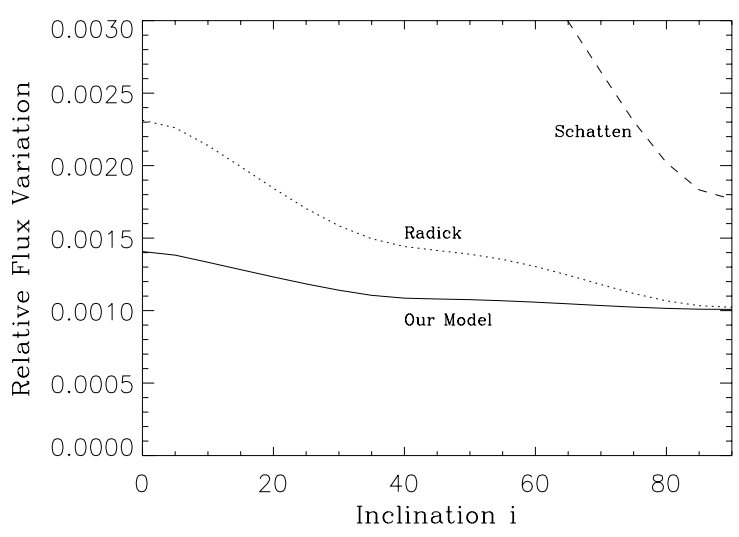

Fig. 10. Comparison of the inclination effect for the flux integrated over the total wavelength range predicted by our model and under the assumptions of R98 and S93.

the chromospheric variability of Sun-like stars may have been systematically underestimated by up to a factor of 2 . However, the averaged Ca II flux depends only marginally on the inclination and should be a reliable measure of the chromospheric activity.

Although we have taken care to keep the parameters of our model realistic, constraining them wherever possible by observations, some uncertainties remain. Firstly, the work of Topka et al. (1997) and Ortiz et al. (2000) suggests that a single facular model may not be sufficient to describe the brightness signature of both active region faculae and active network features (whose surface area coverage also changes somewhat over the activity cycle). Secondly, we have only considered G2V stars whereas the sample investigated by $\mathrm{R} 98$ exhibits a considerable range in spectral types. Stars with different spectral types exhibit different spectral variability. Also, it is unclear if the ratio between spots and faculae on such stars is exactly the same as on the Sun. Thirdly, we have not taken into account a possible change in the brightness of the poles over the solar cycle. At activity minimum, when the Sun's global magnetic field is closest to a north-south dipole, there is an excess of magnetic flux at the poles. To what extent this influences the irradiance variations at different inclinations is unknown.
Acknowledgements. We would like to thank A. C. Cameron for the use of his Doppler imaging code. Y. C. Unruh would also like to acknowledge support through the Fond zur Förderung der wissenschaftlichen Forschung under grant S7302-AST.

\section{References}

Cameron, A. C. 1997, MNRAS, 287, 556

Chapman, G. A., Cookson, A. M., \& Dobias, J. J. 1997, ApJ, 482,541

Fligge, M., \& Solanki, S. K. 2000, J. Geophys. Res., 27, 2157

Fligge, M., Solanki, S. K., Meunier, N., \& Unruh, Y. C. 2000a, ESA SP-463, 117

Fligge, M., Solanki, S. K., \& Unruh, Y. C. 2000b, A\&A, 353, 380

Fligge, M., Solanki, S. K., Unruh, Y. C., Froehlich, C., \& Wehrli, C. 1998, A\&A, 335, 709

Fontenla, J. M., Avrett, E. H., \& Loeser, R. 1993, ApJ, 406, 319

Foukal, P., \& Lean, J. 1988, ApJ, 328, 347

Fröhlich, C. 2000, Space Sci. Rev., 94, 15

Henry, G. W. 1999, PASP, 111, 845

Kurucz, R. L. 1992, Rev. Mex. Astron. Astrofis., 23, 45

Kyle, H. L., Hoyt, D. V., \& Hickey, J. R. 1994, 152, 9

Lockwood, G. W., Skiff, B. A., Baliunas, S. L., \& Radick, R. R. 1992, Nature, 360, 653

Lockwood, G. W., Skiff, B. A., \& Radick, R. R. 1997, ApJ, 485,789

Ortiz, A., Solanki, S. K., Fligge, M., Domingo, V., \& Sanahuja, B. 2000, ESA SP-463, 399

Radick, R. R., Lockwood, G. W., Skiff, B. A., \& Baliunas, S. L. 1998, ApJS, 118, 239

Schatten, K. H. 1993, J. Geophys. Res., 98, 18907

Schrijver, C. J., Cote, J., Zwaan, C., \& Saar, S. 1989, ApJ, 337,964

Skumanich, A., Lean, J. L., Livingston, W. C., \& White, O. R. 1984, ApJ, 282, 776

Skumanich, A., Smythe, C., \& Frazier, E. N. 1975, ApJ, 200, 747

Sofia, S., Schatten, K., \& Oster, L. 1982, Sol. Phys., 80, 87

Topka, K. P., Tarbell, T. D., \& Title, A. M. 1997, ApJ, 484, 479

Unruh, Y. C., Solanki, S. K., \& Fligge, M. 1999, A\&A, 345, 635

White, O. R., Livingston, W. C., Keil, S. L., \& Henry, T. W. 1998, in Synoptic Solar Physics, ASP Conf. Ser., 140, 293

Wilson, O. C. 1978, ApJ, 226, 379 